\title{
SHOCK BREAKOUT AND EARLY LIGHT CURVES OF TYPE II-P SUPERNOVAE OBSERVED WITH KEPLER
}

\author{
P. M. Garnavich ${ }^{1}$, B. E. Tucker ${ }^{2,3}$, A. Rest $^{4}$, E. J. Shaya ${ }^{5}$, R. P. Olling ${ }^{5}$, D Kasen ${ }^{3,6}$, and A. Villar ${ }^{7}$ \\ ${ }^{1}$ Department of Physics, University of Notre Dame, 225 Nieuwland Science Hall, Notre Dame, IN, 46556-5670, USA \\ ${ }^{2}$ The Research School of Astronomy and Astrophysics, Australian National University, Mount Stromlo Observatory, \\ via Cotter Road, Weston Creek, ACT 2611, Australia \\ ${ }^{3}$ Department of Physics and Astronomy, University of California, Berkeley, CA 94720-3411, USA \\ ${ }_{5}^{4}$ Space Telescope Science Institute, 3700 San Martin Drive, Baltimore, MD 21218, USA \\ 5 Astronomy Department, University of Maryland, College Park, MD 20742-2421, USA \\ ${ }^{6}$ Lawrence Berkeley National Laboratory, 1 Cyclotron Road, Berkeley, California 94720, USA \\ ${ }^{7}$ Harvard-Smithsonian Center for Astrophysics, 60 Garden Street, Cambridge, MA 02138, USA \\ Received 2015 July 31; accepted 2016 January 18; published 2016 March 14
}

\begin{abstract}
We discovered two transient events in the Kepler field with light curves that strongly suggest they are type II-P supernovae (SNe II-P). Using the fast cadence of the Kepler observations we precisely estimate the rise time to maximum for KSN2011a and KSN2011d as $10.5 \pm 0.4$ and $13.3 \pm 0.4$ rest-frame days, respectively. Based on fits to idealized analytic models, we find the progenitor radius of KSN2011a $\left(280 \pm 20 R_{\odot}\right)$ to be significantly smaller than that for KSN2011d $\left(490 \pm 20 R_{\odot}\right)$, but both have similar explosion energies of $2.0 \pm 0.3 \times 10^{51} \mathrm{erg}$. The rising light curve of KSN2011d is an excellent match to that predicted by simple models of exploding red supergiants (RSG). However, the early rise of KSN2011a is faster than the models predict, possibly due to the supernova shock wave moving into pre-existing wind or mass-loss from the RSG. A mass-loss rate of $10^{-4} M_{\odot} \mathrm{yr}^{-1}$ from the RSG can explain the fast rise without impacting the optical flux at maximum light or the shape of the post-maximum light curve. No shock breakout emission is seen in KSN2011a, but this is likely due to the circumstellar interaction suspected in the fast rising light curve. The early light curve of KSN2011d does show excess emission consistent with model predictions of a shock breakout. This is the first optical detection of a shock breakout from a SNe II-P.
\end{abstract}

Key words: shock waves - stars: mass-loss - supernovae: general - supernovae: individual (KSN2011a, KSN2011d, SN1999ig)

\section{INTRODUCTION}

Type II-P supernovae (SNe II-P) result from the corecollapse of supergiant stars with significant hydrogen envelopes. Stars exceeding about eight times the mass of the Sun evolve to produce an iron core that cannot be supported against gravity and the resulting collapse drives a shock wave that disrupts the star. Details of how the gravitational energy is converted to an explosion driven by an outward propagating shock are not completely understood, and may require core accretion instabilities (Blondin et al. 2003) or additional energy deposition by neutrinos (Bethe \& Wilson 1985). However, there is clear observational evidence from archival studies of nearby SNe II-P that their progenitors are supergiant stars with radii several hundred times that of the Sun (see Smartt 2015, for a review).

When the shock generated by the core-collapse reaches the surface of the star, a bright flash of hard radiation is expected (Falk 1978; Klein \& Chevalier 1978). The timescale for shock breakout is roughly the time it takes light to traverse the stellar radius (Nakar \& Sari 2010). For typical supergiants this timescale is less than an hour, meaning shock breakouts are very difficult to observe directly. Strong indirect evidence for a hard radiation from a breakout in a SNe II-P comes from the ionized circumstellar rings around SN 1987A (Fransson et al. 1989). Fortuitously, shock breakouts in two SNe II-P have recently been directly detected using the ultraviolet capabilities of the GALEX satellite (Schawinski et al. 2008; Gezari et al. 2015). Both of these UV observed shock breakouts lasted significantly longer than an hour, implying that either the supergiant has an extremely large radius or the presence of circumstellar material prolonged the UV emission (Ofek et al. 2010; Chevalier \& Irwin 2011).

After shock breakout the bulk of the exploded star expands and the effective temperature drops. The competition between the increasing size of the photosphere and the falling temperature determines the early light curve on the timescale of a few days. For simple assumptions of a fixed density profile and constant opacity dominated by electron scattering, the photospheric radius and temperature can be parameterized by the progenitor mass, radius, and explosion energy (Nakar \& Sari 2010; Rabinak \& Waxman 2011). Approaching maximum light these simple assumptions break down, and detailed modeling is required to account for opacity variations with depth and wavelength (e.g., Dessart et al. 2013). Thus observations of the early light curve are important for constraining progenitor properties while relying on a minimum of assumptions.

A recent study of the rise time of SNe II-P has suggested that their progenitors are typically smaller than supergiants cataloged in the Galaxy (Gonzalez-Gaitan et al. 2015). Some of this discrepancy could be due to selection bias in the catalogs since larger stars at a given temperature are easier to detect than their smaller cousins. Still, it may be that progenitors of SNe II-P are more compact than typically thought, or circumstellar interaction makes the rise time appear shorter than expected.

Here, we present Kepler Space Telescope observations of two SNe II-P candidates (see Table 1). The light curves began before explosion and were obtained with unprecedented 30 minute cadence and good photometric precision. While these Kepler observations have several advantages over other 
Table 1

Kepler Type II-P Supernova Candidates

\begin{tabular}{lccccrr}
\hline \hline Name $^{\mathrm{a}}$ & $\begin{array}{c}\text { Host } \\
\text { KIC }^{\mathrm{b}}\end{array}$ & $\begin{array}{c}\text { SN } \\
\text { Type }\end{array}$ & $\begin{array}{c}\text { Redshift } \\
(z)\end{array}$ & $\begin{array}{c}\text { MW } A_{V} \\
(\mathrm{mag})\end{array}$ & $\begin{array}{c}\text { Peak Kp } \\
(\mathrm{mag})\end{array}$ & $\begin{array}{r}\text { Date of Breakout } \\
(\text { BJD-2454833.0) }\end{array}$ \\
\hline KSN 2011a & 08480662 & IIP & 0.051 & 0.194 & $19.66 \pm 0.03$ & $\begin{array}{r}\text { Rise Time } \\
(\text { days })\end{array}$ \\
KSN 2011d & 10649106 & IIP & 0.087 & 0.243 & $20.23 \pm 0.04$ & $1034.15 \pm 0.05$ \\
\hline
\end{tabular}

Notes.

${ }^{a}$ Kepler SuperNovae (KSN) 2011b, 2011c and 2012a were published in Olling et al. (2015).

${ }^{\mathrm{b}}$ Kepler Input Catalog (Brown et al. 2011).

${ }^{c}$ Not corrected for extinction.

studies of SNe II-P, the red-sensitive Kepler bandpass is not ideal for detecting shock breakout radiation. Furthermore, the way Kepler data was taken made it difficult to study transient events in "real time," so little is known about these supernovae other than their exquisite light curves, which are analyzed in their entirety by B. E. Tucker et al. (2016, in preparation).

\section{OBSERVATIONS}

While the primary goal of the Kepler mission (Haas et al. 2010) was to find and study extra-solar planets, it also provided nearly continuous observations of many galaxies. Several Kepler guest observer projects monitored about 500 galaxies at a 30 minute cadence to look for brightness variations in their centers indicative of an active galactic nucleus or to specifically search for supernovae. Targets were selected from the 2MASS extended source catalog (NASA/IPAC IRSA) and the NASA/ IPAC Extragalactic Database (NED). Typically, galaxies were monitored for two to three years, leading to the discovery of three SNe Ia (Olling et al. 2015), one probable type IIn event (P. M. Garnavich et al. 2016, in preparation), and the supernovae presented here. Unfortunately the timescale for the release of Kepler data meant that follow-up of the events was not possible from ground-based observatories. We did obtain spectra of the host galaxies that provided redshifts of the supernovae and information on the environment around the progenitors (B. E. Tucker et al. 2016, in preparation).

On a timescale of minutes to hours, Kepler provides photometric precision of a few parts per million for bright sources. However, on longer timescales, various systematic effects considerably reduce the precision of the standard Kepler products. For example, the Kepler observations were organized in three-month segments labeled quarters Q0-Q16. Each quarter that the spacecraft rotated to keep the Sun on the solar panels resulted in the targets shifting to different detectors. About once per month, the spacecraft goes through a pointing maneuver to downlink the data to Earth. Significant sensitivity variations in the pipeline light curves after repointing maneuvers are removed through special processing. Details of our Kepler reduction procedures can be found in Olling et al. (2015) and Shaya et al. (2015).

\section{LIGHT CURVES}

KSN 2011a was discovered in the galaxy KIC8480662, which is a bright 2MASS galaxy at a redshift of $z=0.051$ (B. E. Tucker et al. 2016, in preparation). The Kepler light curve shows a fast rise, a broad maximum followed by a long plateau (see Figure 1). Finally there is a rapid decay followed by an exponential decline. The light curve is characteristic of $\mathrm{SNe}$ II-P.

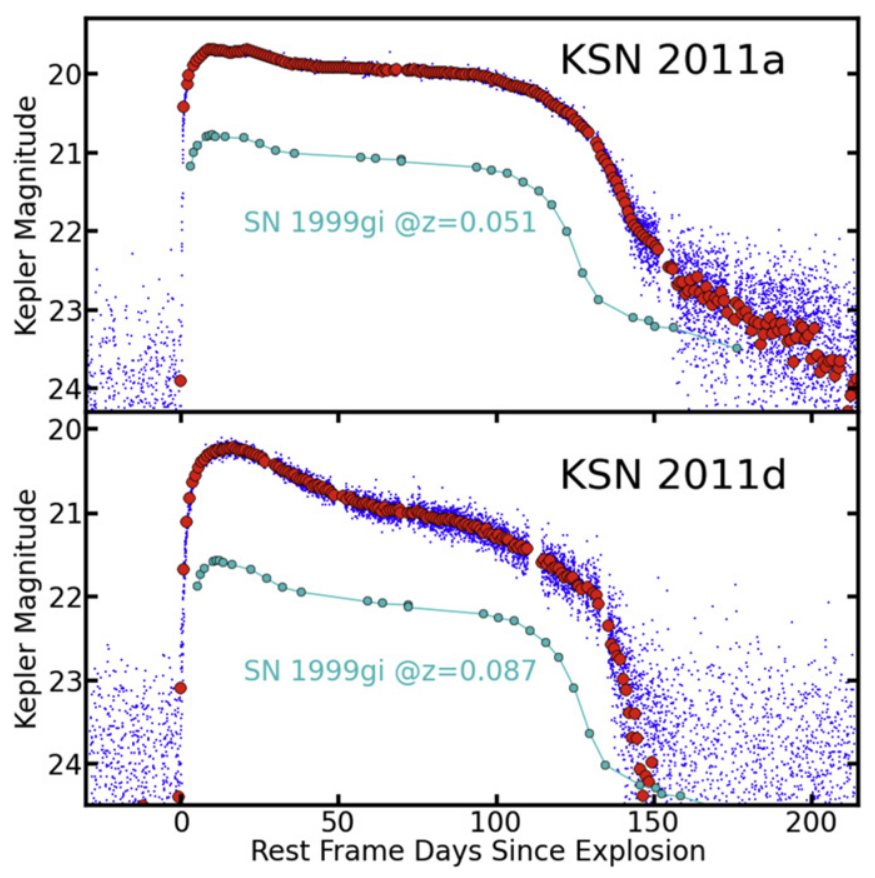

Figure 1. The Kepler light curves of KSN 2011a (top) and KSN 2011d (bottom). The blue points are magnitudes estimated from the standard Kepler 30 minute cadence while the large red symbols show 1 day medians. The small symbols connected by a line display the light curve of the prototypical type IIP SN 1999gi (Leonard et al. 2002) after correction to the redshift of the Kepler events. The initial rise of KSN2011a is clearly faster than KSN2011d based on the number of red points (1 day median) before maximum light.

KSN 2011d was discovered in the galaxy KIC10649106, which is also a 2MASS cataloged galaxy at a redshift of $z=0.087$ (B. E. Tucker et al. 2016, in preparation). Its light curve also shows a fast rise, a broad maximum, and then a slow decay before falling off the "plateau" after 130 days. KSN2011d appears to fade faster on the plateau than KSN2011a, but part of that can be attributed to the higher redshift which means the bandpass contains bluer light that fades more quickly in SNe II-P. A detailed analysis of the full light curves can be found in B. E. Tucker et al. (2016, in preparation).

These Kepler supernovae light curves are very similar to several well-observed SNe II-P events such as SN1999em (Suntzeff, private com.), SN1999gi (Leonard et al. 2002), and SN2012aw (Bose et al. 2013). The Kepler supernovae are at significantly higher redshifts than these local events, so $k$ corrections are important, but there is no color information for the Kepler events. Therefore, we use the BVRI magnitudes of 


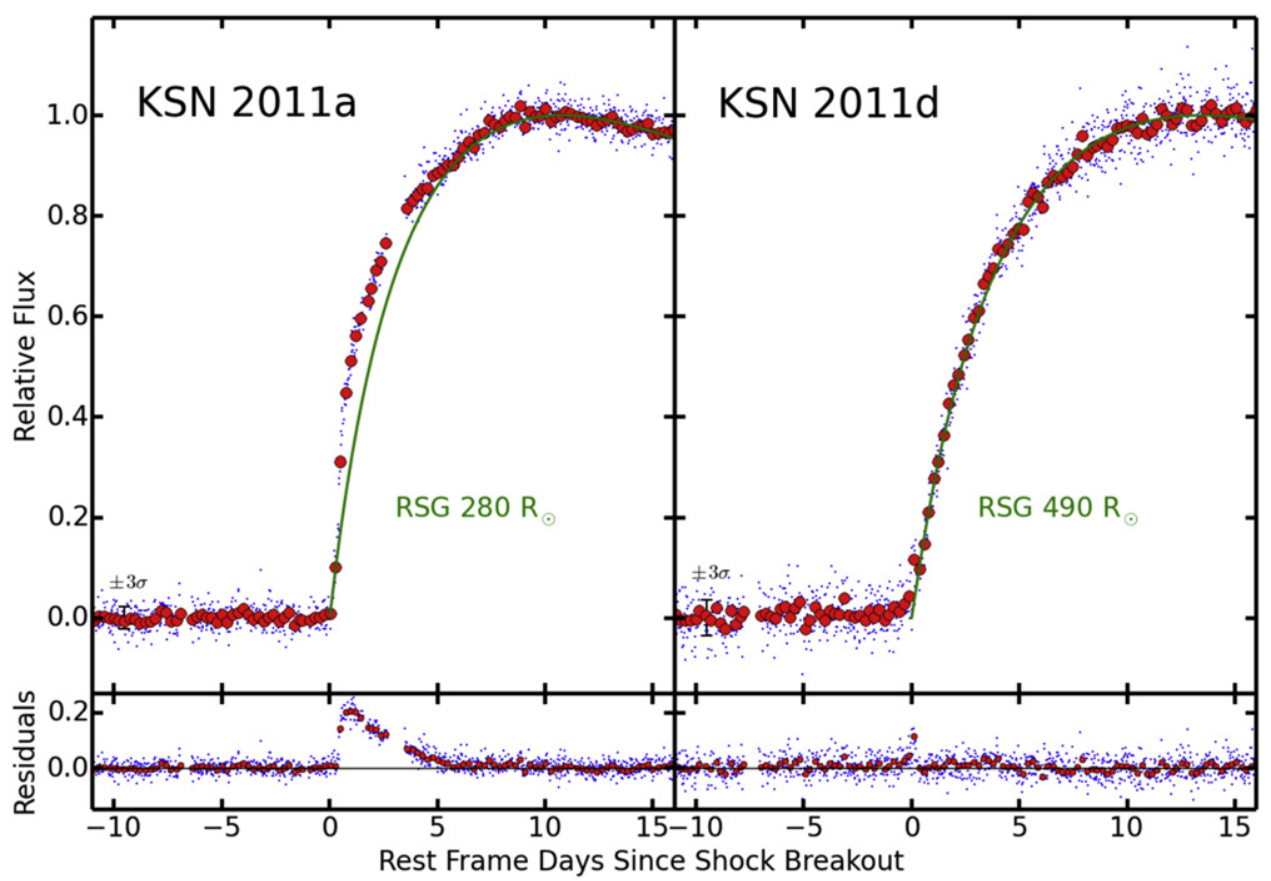

Figure 2. The early light curves of the two Kepler SNe II-P. Blue dots are individual Kepler flux measurements with a 30 minute cadence and the red symbols are 6 hr medians. The $x$-axis shows the redshift corrected time since shock breakout estimated from the model fit. Note that a shock traversing a red supergiant can take about a day to reach the surface, so we cannot measure the time of core-collapse. Right: the light curve for KSN2011d with a model fit assuming a progenitor radius of $490 R_{\odot}$. An error bar at -10 days indicates the $3 \sigma$ uncertainty on the median points. The lower panel shows the residuals to the fit. Left: the light curve of KSN2011a with a model fit using a progenitor radius of $280 R_{\odot}$. The model cannot match the fast rise early in the light curve and still fit the time of maximum. The lower panel shows significant residuals that decay on a timescale of 5 days.

the nearby supernovae to correct them to the Kepler observed frame.

For the nearby supernovae we create a spectral energy distribution (SED) for each epoch observed in multiple filters. Missing filters are interpolated from adjacent epochs. The SEDs are corrected for Milky Way extinction using Schlafly \& Finkbeiner (2011). The SED is corrected to the redshift of the Kepler events, convolved with the Kepler bandpass, and the result is integrated to give the total photon flux. The result is also reddened to match the Milky Way extinction in the direction of the Kepler supernova. Kepler magnitudes are in the $\mathrm{AB}$ system, so the Kepler bandpass is convolved with a spectrum with constant $F_{\nu}=3631 \mathrm{Jy}$ and integrated to determine the magnitude zeropoint.

For comparison, Figure 1 displays the light curve of the wellobserved local event SN 1999gi after correction to the redshifts of the Kepler supernovae. SN 1999gi was a slightly fainter than typical SNe II-P (Bose et al. 2013), and ignoring unknown host extinction, it is over a magnitude fainter than these two Kepler supernovae. Still, the shape of the light curve and length on the plateau make the SNe II-P classifications of KSN2011a and KSN2011d very solid.

\section{ANALYSIS}

\subsection{Rise to Maximum}

The rapid cadence of the Kepler observations provides a unique window into the early rise of supernovae. In particular, SNe II-P have rise times on the order of a week and are difficult to capture in typical ground-based surveys (e.g., GonzalezGaitan et al. 2015). In Figure 2, we show the Kepler light curves beginning several days before explosion and ending soon after maximum light. There are approximately 500 individual photometric measurements between the initial brightening and maximum light. But these events are at significant distance, so we have combined the Kepler cadence into $6 \mathrm{hr}$ median bins to improve the signal-to-noise ratio of the light curves.

To fit the pre-maximum rise of the Kepler events, we calculated a grid of light curves using the Rabinak \& Waxman (2011) RSG model. The RSG model assumes a power-law density structure with an index $n=3 / 2$. We vary the progenitor radius and explosion energy, keeping the stellar mass at $15 M_{\odot}$, which is typical for core-collapse supernovae (Smartt 2015). We assume fully ionized hydrogen envelopes $\left(\kappa=0.34 \mathrm{~cm}^{2} \mathrm{~g}^{-1}\right)$ and set the normalization of the ejecta density to $f_{p}=0.1$, although the results are not sensitive to this parameter. The Rabinak \& Waxman (2011) model allows us to calculate the temperature and radius of the expanding photosphere as a function of time. For each epoch we construct a blackbody corrected to the redshift and distance of the Kepler supernova and multiply the spectrum by the Kepler sensitivity function. We then integrate and normalize the resulting flux using the zeropoint calculated in the $\mathrm{AB}$ magnitude system. For each pair of initial radius and explosion energy we have a light curve from which we derive a rise time and peak magnitude.

The observed rise time (and uncertainty) defines a band in the radius versus energy plane that is nearly horizontal, while the observed magnitude (and error) defines a band that cuts diagonally across the parameters of interest. The results for the two Kepler supernovae are presented in Figure 3. The intersection of the two bands is a consistent fit to the rise time and peak brightness (corrected for Milky Way extinction) and tightly constrains the derived quantities of progenitor radius and explosion energy. We also created model grids for 

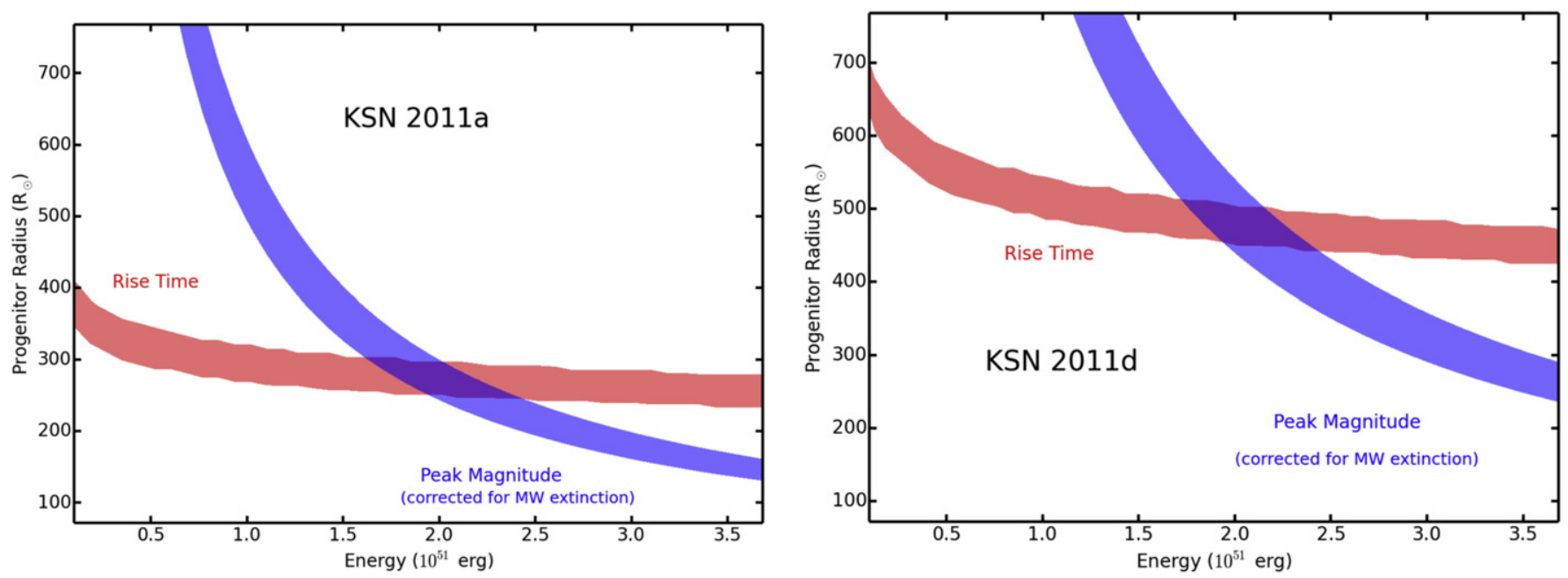

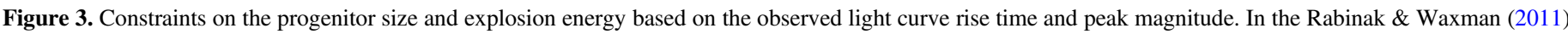

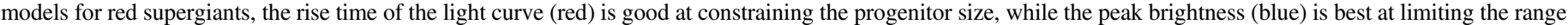

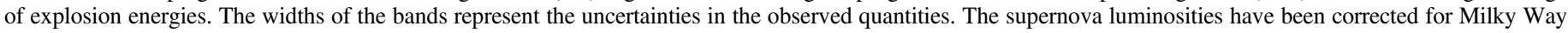

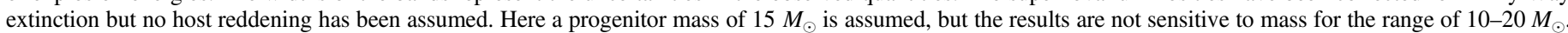

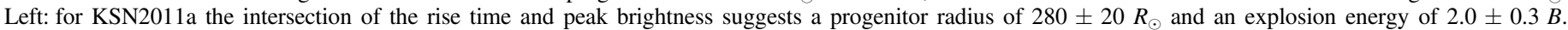
Right: the Kepler observations of KSN2011d suggest a larger progenitor at $490 \pm 20 \mathrm{R}_{\odot}$ but a similar explosion energy of $2.0 \pm 0.3 B$.

progenitor masses of 10 and $20 M_{\odot}$, but the resulting parameters did not differ significantly from the $15 M_{\odot}$ calculations.

The model light curves that best match the observed rise time and peak magnitude are then fit to the actual light curves by minimizing the residuals between the model and data. From this a new time of explosion is estimated and an improved rise time is calculated. This iterative method converges quickly and the resulting fits are shown in Figure 2.

For KSN2011d, the Rabinak \& Waxman (2011) model with a progenitor radius of $490 R_{\odot}$ and explosion energy ${ }^{8}$ of $2 \pm 0.3 B$ not only matches the rise time and peak magnitude, but the Rabinak \& Waxman (2011) model predicts the overall shape of the rise very well. The $\chi^{2}$ parameter of the best fit model is 1532 for 1142 free parameters or a reduced $\chi_{\nu}^{2}=1.34$.

The best fit rise time for KSN2011a is $280 \pm 20 R_{\odot}$ and the explosion energy is also $2 \pm 0.3 \mathrm{~B}$. Kasen \& Woosley (2009) found that only a few nearby SNe II-P events had energies larger than $1.5 \mathrm{~B}$, so finding two Kepler supernovae with explosion energies of $2 B$ is surprising. But the Kepler supernova search is likely biased toward discovering luminous events near the limiting magnitude of the survey and these will tend to have higher explosion energies than supernovae found in volume-limited searches of nearby events.

No correction has been made for possible host extinction because colors are not measured for these events. The explosion energy will be the parameter most affected by our uncertainty in host reddening and in our analysis we are actually estimating its lower limit. Poznanski et al. (2009) estimated the color excess for forty SNe II-P events and from that sample we infer a median visual extinction of $0.80 \mathrm{mag}$. We note that this sample of relatively nearby SNe II-P may not be representative of the magnitude-limited Kepler discoveries, but it does suggest significant extinction is not unusual for $\mathrm{SNe}$ II-P.

$81 B=1$ foe $=10^{51} \mathrm{erg}$.
The shape of the KSN2011a light curve is not as well fit by the Rabinak \& Waxman (2011) prediction (left panel of Figure 2) even when the times to maximum and peak magnitude are well matched. In the first five days the KSN2011a light curve rises significantly faster than the model even though the same physics resulted in an excellent fit to the KSN2011d light curve. Assuming a smaller progenitor radius does make the model rise faster, but that model will then peak much earlier than the observed 10.5 days. Smaller assumed radii also produce a poor fit near maximum light for blue supergiant (BSG) models which differ from RSG in their density profile.

The rapid rise in KSN2011a and the "extra light" above the Rabinak \& Waxman (2011) model photosphere suggests the supernova shock continued to propagate into circumstellar material, allowing it to convert more kinetic energy into luminosity and diffuse the shock breakout over a longer time. Strong circumstellar interaction has been successful in explaining very luminous events (Ofek et al. 2010; Chevalier \& Irwin 2011), but progenitors with low mass-loss rates may also see their early light curves enhanced with a weak shock interaction (Moriya et al. 2011).

Moriya et al. (2011) calculations show that mass-loss rates of the order of $10^{-4} M_{\odot} \mathrm{yr}^{-1}$ will cause the early light curve to rise faster than a bare RSG, while not strongly affecting the optical peak luminosity or the light curve during the plateau. Mass-loss rates less than $10^{-4} M_{\odot} \mathrm{yr}^{-1}$ mean that the circumstellar medium near the progenitor radius is too low-density to become optically thick when the shock hits it, so the presence of the wind would have no significant impact on the light curve even during the early rise. We therefore expect that the mild interaction seen in KSN2011a is due to a mass-loss rate just above the $10^{-4} M_{\odot} \mathrm{yr}^{-1}$ threshold.

\subsection{Shock Breakout}

\subsubsection{KSN2011a}

The fast cadence and continuous coverage of Kepler should, in principle, allow us to see the shock breakouts in these $\mathrm{SNe}$ 


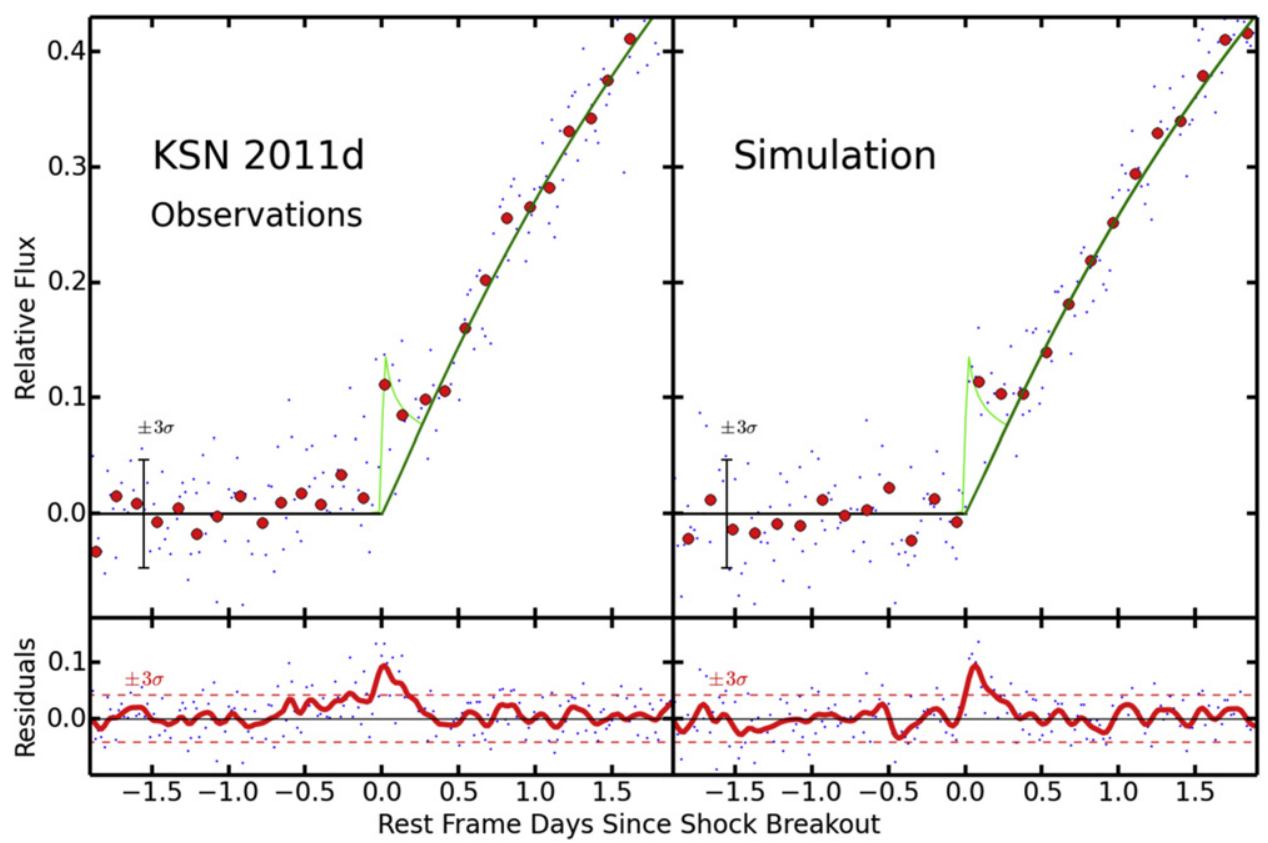

Figure 4. Left: the Kepler light curve of KSN2011d focused on the time expected for shock breakout. The blue dots are individual Kepler measurements and the red symbols show $3.5 \mathrm{hr}$ medians of the Kepler data. An error bar at -1.5 days indicates the $3 \sigma$ uncertainty on the median points. The green line shows the best fit photospheric model light curve. The lower panel displays the residuals between the observations and the model fit. The thick red line is a Gaussian smoothed residual light curve using a full-width at half-maxmimum of two hours. The dashed red lines indicate $3 \sigma$ deviations of the Gaussian smoothed curve. The residual at the time expected for shock breakout is more than $5 \sigma$, implying that the feature is unlikely to be a random fluctuation. Right: a simulated light curve created using the statistical properties of the Kepler photometry and the best fit photospheric model. In addition, a Nakar \& Sari (2010) shock breakout model (light green line) for an explosion energy of $2 B$ and radius of $490 R_{\odot}$ is compared with both the data and simulation.

II-P. In the case of KSN2011a where we suspect circumstellar interaction, the shock breakout was likely reprocessed over the diffusion time in the optically thick wind. The shock continued into the wind and converted additional kinetic energy into luminosity that we see as excess flux during the photospheric rise. The peak absolute Kepler magnitude from the circumstellar interaction is $M_{\mathrm{Kp}}=-15.5 \mathrm{mag}$, but much of the total energy is likely emitted at shorter wavelengths.

\subsubsection{KSN2011d}

In the KSN2011d light curve (Figure 2), there is a single $6 \mathrm{hr}$ median flux point that deviates from the light curve model by 4 standard deviations $(\sigma)$ at the time expected for shock breakout. A close-up of this time period is shown in Figure 4 using a binning width of $3.5 \mathrm{hr}$. Extrapolating the Rabinak \& Waxman (2011) photospheric model to zero flux predicts shock breakout at $t_{0}=2455873.75 \pm 0.05 \mathrm{BJD}$ which corresponds to the time of the largest deviation from the model.

When we subtract the best fit photosphere model for KSN2011d there remains seven Kepler photometric observations within five hours of $t_{0}$ that are $3 \sigma$ or more above zero (lower panel in Figure 4). To avoid bias that might come from dividing the data into bins, we have smoothed the light curve residuals using a Gaussian with a full-width at half-maximum (FWHM) of $2 \mathrm{hr}$. There is a clear $6 \sigma$ peak at the time expected for breakout and we conclude that this is, indeed, the shock breakout from KSN2011d. The shock breakout flux is $12 \%$ of the peak flux of the supernova, corresponding to a Kepler magnitude of $22.3 \pm 0.2$ after correcting for Milky Way extinction.

In the Nakar \& Sari (2010) shock breakout model, the initial rise is the result of diffusion of the shock emission before the shock reaches the stellar surface and is only of the order of five minutes. This is too short a time for even the Kepler cadence, so the rise to shock breakout is unresolved. After shock breakout the flux decay follows a $t^{-4 / 3}$ power law in time until the expanding photosphere dominates the luminosity. This decay is relatively slow and allows the breakout to remain detectable for several hours. From the Nakar \& Sari (2010) formulation, we can estimate the ratio between the peak flux from the shock breakout, $F_{\mathrm{SB}}$, and the maximum photospheric flux, $F_{\max }$, which we approximate as the brightness 10 days after explosion. Using the ratio between the shock peak and photosphere maximum is particularly useful since it eliminates the uncertainty caused by distance and dust extinction. In the rest-frame optical (5500 $\AA$ ) the flux ratio is

$$
F_{\mathrm{SB}} / F_{\max }=0.25 M_{15}^{0.54} R_{500}^{0.73} E_{51}^{-0.64}
$$

where $M_{15}$ is the progenitor mass in units of $15 M_{\odot}, R_{500}$ is the progenitor radius in units of $500 R_{\odot}$, and $E_{51}$ is the explosion energy in units of $10^{51} \mathrm{erg}$. So we expect the shock breakout in a typical RSG to peak at about $25 \%$ of visual brightness of the supernova at maximum.

Applying the Nakar \& Sari (2010) model to KSN2011d (radius of $490 R_{\odot}$, energy of $2 B$ and a progenitor mass of $\left.15 M_{\odot}\right)$, predicts a breakout temperature of $2 \times 10^{5} \mathrm{~K}$, and Equation (1) gives $F_{\mathrm{SB}} / F_{\max }=0.16$, meaning the shock should be 2 mag fainter in the optical than the supernova at maximum. The Kepler 30 minute cadence will smooth the sharp peak of the breakout and lower the maximum by $20 \%$ so we expect the ratio to be $F_{\mathrm{SB}} / F_{\max }=0.13$. The excess flux seen in Figure 4 peaks at a relative flux of $0.12 \pm 0.2$ and is consistent with the Nakar \& Sari (2010) prediction. 
We use a blackbody spectrum to extrapolate the shock breakout flux down to the optical and this is likely a poor approximation. So it is surprising that the semi-analytic model of Nakar \& Sari (2010) works so well in matching the observed breakout. Tominaga et al. (2011) calculated realistic spectra at breakout for a variety of RSG models. While color temperatures and integrated luminosities varied greatly, the peak optical flux at breakout was fairly consistent: between $2 \times 10^{37}$ and $1 \times 10^{38} \mathrm{erg} \mathrm{s}^{-1} \AA^{-1}$, corresponding to absolute magnitudes between -14.2 and $-15.9 \mathrm{mag}$. The Tominaga et al. (2011) model for a $15 M_{\odot}, 1 B$ and $500 R_{\odot}$ RSG predicts $^{9}$ a peak at $M_{\mathrm{Kp}}=-14.4 \mathrm{mag}$. Doubling the explosion energy would brighten the breakout by about $0.2 \mathrm{mag}$, yielding a luminosity of $M_{\mathrm{Kp}}=-14.6 \mathrm{mag}$. The observed shock breakout from KSN2011d is $M_{\mathrm{Kp}}=-15.6 \pm 0.3 \mathrm{mag}$ (after correction for Milky Way extinction; assuming $H_{0}=70 \mathrm{~km} \mathrm{~s}^{-1} \mathrm{Mpc}^{-1}$ ). Overall, the models do an excellent job in predicting the optical brightness of the shock breakout in KSN2011d.

We have simulated the light curve of KSN2011d using the statistical properties of the Kepler photometry based on 100 days prior to the supernova detection (right panel in Figure 4). The photospheric rise of the light curve uses the Rabinak \& Waxman (2011) model and the shock breakout uses the Nakar \& Sari (2010) model with our best fit parameters. Assuming Gaussian statistics, we created a Monte Carlo simulation of the models sampled at the Kepler cadence. The simulation matches the observed light curve extremely well and we again conclude that we have detected the shock breakout in KSN2011d.

\subsubsection{Radiative Precursor?}

In the $12 \mathrm{hr}$ before shock breakout only 2 of the 24 Kepler observations fall below the median pre-supernova flux. That is, the light curve of KSN2011d shows a possible slow rise in brightness starting 0.5 days before breakout. This is intriguing, as core-collapse likely occurred nearly a day before shock breakout (Chevalier \& Irwin 2011). The shock travels more slowly than photons diffuse through the RSG envelope, allowing evidence for the shock to reach the surface before breakout. However, Nakar \& Sari (2010) predicted the shock energy would leak out through diffusion on a timescale of only five minutes for a RSG. In contrast, Schawinski et al. (2008) suggested a "radiative precursor" due to photon diffusion could begin hours before shock breakout and there is some evidence for a precursor seen in the GALEX detection of a SNe II-P five hours before the peak breakout emission.

While it is tantalizing to claim precursor emission in KSN2011d, the smoothed flux remains at $3 \sigma$ or less from median value, so this detection is not definitive. More observations of SNe II-P with extremely fast cadence are needed to determine the diffusion timescale before shock breakout.

\section{CONCLUSION}

We discovered two transient events in the Kepler field with light curves that strongly suggest they are SNe II-P events. From the fast cadence of the Kepler observations we determine the time the supernova shock reached the surface of the progenitor with a precision of better than 0.1 days. We find the

\footnotetext{
$9 \mathrm{Kp} \approx 0.2 g+0.8 r$ where $g$ and $r$ are SDSS magnitudes (Kepler Calibration webpage).
}

rise time to maximum was $10.5 \pm 0.4$ rest-frame days for KSN2011a and $13.3 \pm 0.4$ days for KSN2011d. From the rise times combined with their peak luminosities (not corrected for host extinction), we estimate the progenitor radius of KSN2011a $\left(280 R_{\odot}\right)$ to be significantly smaller than that for KSN2011d (490 $\left.R_{\odot}\right)$, but both have similar explosion energies of $2 B$.

Our directly measured rise-times for both Kepler events are many standard deviations larger than the median rise-time of $\mathrm{SNe}$ II-P estimated from an ensemble of light curves by Gonzalez-Gaitan et al. (2015). This difference results from the variation of rise-time with effective wavelength. Gonzalez-Gaitan et al. (2015) referenced their rise-time to the SDSS- $g$ band while light curves peak several days later in the redder Keplerbandpass. The median radius of the progenitors estimated by Gonzalez-Gaitan et al. (2015) is $320 R_{\odot}$, which is comparable to the radii we infer from the Kepler data. As with the Gonzalez-Gaitan et al. (2015) estimates, radii measured from SNe II-P light curves tend to be at the low end of the distribution of radii estimated for RSG directly observed in the Milky Way (Levesque et al. 2005).

The rising light curve of KSN2011d is an excellent match to that predicted by the Rabinak \& Waxman (2011) models for RSG. However, the Rabinak \& Waxman (2011) models underestimate the brightness of KSN2011a during the first five days and we suggest that the additional flux is due to the supernova shock moving into a pre-existing wind or mass-loss from the RSG. Moriya et al. (2011) has shown that a mass-loss rate of $10^{-4} M_{\odot} \mathrm{yr}^{-1}$ will steepen the light curve while not strongly impacting the flux at maximum light or the shape of the post-maximum light curve.

No fast shock breakout emission is seen in KSN2011a, but this is likely due to the circumstellar interaction suspected in the early light curve. KSN2011d does show excess emission at the time expected for shock breakout with a brightness of $12 \%$ that of supernova peak in the Kepler band. The timescale and brightness observed for the breakout are consistent with model predictions.

The diversity in the rising light curves of SNe II-P observed by Kepler show that early observations are critical in understanding the progenitors and circumstellar environments of exploding RSG stars.

This research has made use of the NASA/IPAC Extragalactic Database (NED), which is operated by the Jet Propulsion Laboratory, California Institute of Technology, under contract with the National Aeronautics and Space Administration. This research has made use of the NASA/ IPAC Infrared Science Archive, which is operated by the Jet Propulsion Laboratory, California Institute of Technology, under contract with the National Aeronautics and Space Administration. This work was partly supported by Kepler grants NNX12AC89G and NNX11AG95G. D.K. is supported by a DOE office of nuclear physics early career award.

\section{REFERENCES}

Bethe, H. A., \& Wilson, J. R. 1985, ApJ, 295, 14

Blondin, J. M., Mezzacappa, A., \& DeMarino, C. 2003, ApJ, 584, 971

Bose, S., Kumar, B., Sutaria, F., et al. 2013, MNRAS, 433, 1871

Brown, T. M., Latham, D. W., Everett, M. E., \& Esquerdo, G. A. 2011, AJ, 142,112 
Chevalier, R. A., \& Irwin, C. M. 2011, ApJ, 729, 6

Dessart, L., Hillier, D. J., Waldman, R., \& Livne, E. 2013, MNRAS, 433, 1745

Falk, S. W. 1978, ApJ, 225, 133

Fransson, C., Cassatella, A., Gilmozzi, R., et al. 1989, ApJ, 336, 429

Gezari, S., Jones, D. O., Sanders, N. E., et al. 2015, ApJ, 804, 28

Gonzalez-Gaitan, S., Tominaga, N., Molina, J., et al. 2015, arXiv:1505.02988

Haas, M., Batalha, N. M., Bryson, S. T., et al. 2010, ApJ, 713, 115

Kasen, D., \& Woosley, S. E. 2009, ApJ, 703, 2205

Klein, R. I., \& Chevalier, R. A. 1978, ApJ, 223, 109

Leonard, D. C., Filippenko, A. V., Li, W., et al. 2002, AJ, 124, 2490

Levesque, E. M., Massey, P., Olsen, K. A. G., et al. 2005, ApJ, 628, 973
Moriya, T., Tominaga, N., Blinnikov, S. I., Baklanov, P. V., \& Sorokina, E. I. 2011, MNRAS, 415, 199

Nakar, E., \& Sari, R. 2010, ApJ, 725, 904

Ofek, E. O., Rabinak, I., Neill, J. D., et al. 2010, ApJ, 724, 1396

Olling, R., Mushotzky, R., Shaya, E. J., et al. 2015, Natur, 521, 332

Poznanski, D., Butler, N., Filippenko, A. V., et al. 2009, ApJ, 694, 1067

Rabinak, I., \& Waxman, E. 2011, ApJ, 728, 63

Schawinski, K., Justham, S., Wolf, C., et al. 2008, Sci, 321, 223

Schlafly, E. F., \& Finkbeiner, D. P. 2011, ApJ, 737, 103

Shaya, E. J., Olling, R., \& Mushotzky, R. 2015, AJ, 150, 188

Smartt, S. J. 2015, PASP, 32, e016

Tominaga, N., Morokuma, T., Blinnikov, S. I., et al. 2011, ApJS, 193, 20 